\title{
Effect of Ointment Containing Epithelial Growth Factor Compared with Antibacterial Ointment in Avulsive Laceration
}

\author{
Youngwoong Choi (1) \\ Department of Plastic and Reconstructive Surgery, Inje University Sanggye Paik Hospital, Inje University College of Medicine, Seoul, Korea
}

\begin{abstract}
Background: Avulsive lacerations are more likely to cause complications such as necrosis and dehiscence than vertical lacerations. In laceration wounds, topical antimicrobials can be effective because of their antibacterial properties that help eliminate infection or biofilms from the wound bed. However, indiscriminate or habitual use of antibiotics may cause bacterial tolerance. The purpose of this study was to evaluate the effectiveness of an ointment containing epidermal growth factor (EGF), effective in healing wounds involving epithelial and skin cells, compared with an antibiotic ointment for avulsive lacerations.

Methods: We recruited patients who visited the emergency department with avulsive lacerations on their faces. The selected patients were randomly prescribed for dressing either an ofloxacin ointment or EGF ointment after repair. Epidemiologic information of patients, wound assessment (size, depth, and location) and wound healing result assessments (necrosis/dehiscence) were evaluated.

Results: Of the 119 participants, 42 were women and 77 were men. The average length of the lacerations was $2.15 \mathrm{~cm}$ and most lacerations were in the muscle layer. There was no difference in the incidence of dehiscence and necrosis after use of either ointment, but the ratio of the necrotic area was significantly lower in EGF ointment group (antibiotics ointment, 2.33\% and EGF ointment, $0 \% ; P=0.03$ ).

Conclusion: We conclude that the ointment containing EGF can be used to replace the antimicrobial-containing ointment often used to prevent infection, which is in turn followed by skin or soft tissue necrosis.
\end{abstract}

Keywords: Lacerations; Epidermal growth factor; Surgical wound dehiscence; Necrosis; Wound healing

\section{Introduction}

Laceration is one of the most common injuries encountered in the emergency department. Avulsive lacerations, where the wound is at a slant on the skin, are mostly caused by shearing or friction forces. An avulsion laceration is more likely to cause complications such as necrosis and dehiscence than a vertical laceration, where skin damage is vertical on the skin.

Although uncommon in simple lacerations, complications may delay wound healing and cause more scars, resulting in discomfort in daily life. In addition to the established methods of dressing after laceration repair, studies have been undertaken to find an appropriate and effective dressing method to promote wound healing. Epidermal growth factor (EGF) is known to be effective for epidermal proliferation, dermal granulation, and regeneration of injured skin [1]. Moreover, several animal studies have proven the effects of EGF ointments. Since there have been no studies on the effect of EGF on laceration wounds, we aimed to investigate the effect of EGF ointment on avulsion lacerations, which heal slower and have a slightly higher probability of complications such as dehiscence and necrosis.

\section{Original Article}

Received: October 15, 2019

Revised: February 7, 2020

Accepted: February 8, 2020

\section{Corresponding author: Youngwoong Choi, M.D., Ph.D.}

Department of Plastic and Reconstructive Surgery, Inje University Sanggye Paik Hospital, Inje University College of Medicine, 1342 Dongil-ro, Nowon-gu, Seoul 01757, Korea Tel: +82-2-950-1048

Fax: +82-2-932-6373

E-mail: pshero2@naver.com

This is an Open Access article distributed under the terms of the Creative Commons Attribution Non-Commercia License (https://creativecommons org/licenses/by-nc/4 O/) which permits unrestricted non-commercial use, distribution, and reproduction in any medium, provided the original work is properly cited.

C) 2020 Korean Wound Management Society 


\section{Methods}

\section{Subjects and study design}

Patients who visited the emergency department with avulsion lacerations on their faces were recruited in this single-blinded, randomized study. The selected patients were randomly prescribed either an ofloxacin ointment or EGF ointment for dressing after suturing, depending on whether the day the patient was admitted was an odd or even number day. Patients were screened for several conditions; those with hematologic disorder, hematologic malignancy, diabetes mellitus (DM), or immunologic disorder were excluded. Pregnant patients, patients undergoing local hyperbaric oxygen therapy and those who failed to follow-up were also excluded. This study was approved by the Institutional Review Board of Sanggye Paik Hospital (IRB No. 2017-03-009). All subjects provided written informed consent before participating in the study.

The EGF ointment (Saesal Yongo; Daewoong Pharmaceutical Co., Ltd., Seoul, Korea) contains epithelial growth factor for rapid wound healing [2]. Ofloxacin ointment (Effexin Opht ointment; Ildong Pharm., Seoul, Korea) is an antibiotic ointment in the fluoroquinolone family of medications, useful for treating a number of bacterial infections. Either ointment was applied topically over the wound and covered with foam material. Dressing was changed every other day.

In order to measure the size of necrosis, based on clinical photos, the area where necrosis occurred relative to the total area of the wound was assessed. Area of necrosis (\%) was calculated by taking the necrosis area and dividing it by the total wound area, then multiplying it by 100 . Measurements were taken on the photos at the time of stitch removal.

\section{Clinical outcomes evaluation}

The patients were assessed in the emergency department for the size, depth, and location of the wounds on their initial visits after trauma and later in the outpatient department for further wound assessment. Wounds were assessed every other day, and stitches on facial wounds were removed at 5-7 days after initial repair. Physicians evaluated the wound during the healing process. Complete healing was defined as full epithelialization of the wound with absence of discharge. The wounds were examined for dehiscence and infection, and necrosis was evaluated by the percentage of wound surface involved.

\section{Statistical analysis}

The chi-square tests on the statistical analysis used SPSS ver- sion 25.0 (IBM Corp., Armonk, NY, USA).

\section{Results}

A total of 119 patients with facial avulsion lacerations who visited the emergency department for primary repair and the outpatient department for follow-up were enrolled. Patients who did not visit the outpatient department after visiting the

Table 1. Epidemiology of patients with facial avulsion laceration

\begin{tabular}{lc}
\hline Characteristic & No. of patients (\%) \\
\hline Mean age (yr) & 29 \\
Sex & $77(65)$ \\
Male & $42(35)$ \\
Female & 2.15 \\
Average of laceration length (cm) & \\
Depth & $36(30)$ \\
Dermis & $14(12)$ \\
Subcutaneous fat & $61(51)$ \\
Muscle & $8(7)$ \\
Bone & \\
Location & $35(29)$ \\
Forehead & $21(18)$ \\
Eyebrow & $17(14)$ \\
Chin & $14(12)$ \\
Nose & $9(8)$ \\
Upper eyelid & $9(8)$ \\
Cheek & $5(4)$ \\
Lateral canthal area & $4(3)$ \\
Philtrum & $2(2)$ \\
Lower eyelid & $2(2)$ \\
Glabella & $1(1)$ \\
Ear & \\
\hline
\end{tabular}

Cause of injury

Slip down $\quad 86(72)$

Sharp instrument $\quad 8(7)$

Bicycle $6(5)$

Sports $\quad 6(5)$

Physical violence 4 (3)

Fall down 4 (3)

Pedestrian TA 2 (2)

In car TA

Unknown 2(2)

TA, traffic accident. 
emergency room, as well as seven patients with DM were excluded. Of the 119 patients, 42 (35\%) were women and 77 (65\%) were men with ages ranging from 0 to 95 years (mean, 29 years). The average length of the lacerations was $2.15 \mathrm{~cm}$ (range, $0.5-15 \mathrm{~cm}$ ). Muscle layer was the most common depth of the lacerations (51\%), followed by dermis layer (30\%), subcutaneous fat layer (12\%), and bone exposure layer (7\%). The forehead was the most common site of laceration (29\%), followed by eyebrow (18\%) and chin (14\%). Slipping and falling was the most common cause of injury (72\%), while eight patients (7\%) were injured with sharp instruments such as scissors or knives (Table 1). In most patients, stitches were removed within 1 week after suture. The average duration of healing was 6.83 days after suturing.

Ninety patients were assigned to the group with an antibiotic ointment prescription, and 29 patients were assigned to the group with EGF ointment prescription. While dehiscence (without necrosis) occurred in 10 patients (eight from the antibiotic ointment group and two from the EGF ointment group) and necrosis following dehiscence was observed in five patients (all from the antibiotic ointment group), the other patients showed good results without any complications (Table
2). On the chi-square test, there was no significant difference between the incidence of complications in each group. However, there was significant difference between the two groups in the area of necrosis (antibiotics ointment, 2.33\% and EGF ointment, $0 \% ; \mathrm{P}=0.03$ ) for each patient group (Table 3).

Of the patients with complications, 13 received antibiotic ointment and two received EGF ointment. The mean length of laceration in these patients was $3.5 \mathrm{~cm}$, which was longer than the mean length of lacerations in all patients. The average healing

Table 3. Comparison of complications between antibiotic ointment and EGF ointment

\begin{tabular}{lccc}
\hline Complications & $\begin{array}{c}\text { Antibiotic ointment } \\
\text { group }(n=90)\end{array}$ & $\begin{array}{c}\text { EGF ointment } \\
\text { group }(n=29)\end{array}$ & P-value \\
\hline Dehiscence & 8 & 2 & 1.00 \\
Necrosis incidence & 5 & 0 & 0.33 \\
Area of necrosis (\%) & 2.33 & 0 & $0.03^{\mathrm{a})}$ \\
Duration of treatment (day) & 6.59 & 6.91 & 0.32 \\
\hline
\end{tabular}

Values are presented as number or mean.

EGF, epidermal growth factor.

${ }^{a}$ Chi-square test; P-value $<0.05$ were considered to indicate statistical significance.

Table 2. Patients with necrosis or dehiscence

\begin{tabular}{|c|c|c|c|c|c|c|c|c|c|c|c|}
\hline $\begin{array}{c}\text { Patient } \\
\text { no. }\end{array}$ & Sex & Age (yr) & $\begin{array}{l}\text { Ointment } \\
\text { applied }\end{array}$ & Location & Size (cm) & Depth & Cause & $\mathrm{PMH}$ & Complication & $\begin{array}{l}\text { Necrosis } \\
\text { rate (\%) }\end{array}$ & $\begin{array}{c}\text { Duration of } \\
\text { healing } \\
\text { (day) }\end{array}$ \\
\hline 1 & Male & 2 & EGF & Forehead & 1.5 & M & Slip down & - & Dehiscence & - & 14 \\
\hline 2 & Female & 83 & Antibiotic & Forehead & 3.0 & M & Slip down & $\begin{array}{l}\text { Hypertension, } \\
\text { CAOD }\end{array}$ & Dehiscence & - & 9 \\
\hline 3 & Female & 5 & Antibiotic & Philtrum & 1.5 & SC & Slip down & Depression & Necrosis & 50 & 12 \\
\hline 4 & Female & 2 & Antibiotic & Forehead & 1.5 & M & Slip down & - & Dehiscence & - & 10 \\
\hline 5 & Male & 4 & EGF & Forehead & 3.0 & B & Fall down & - & Dehiscence & - & 13 \\
\hline 6 & Male & 47 & Antibiotic & Cheek & 2.0 & $\mathrm{SC}$ & Human trouble & - & Necrosis & 50 & 24 \\
\hline 7 & Male & 52 & Antibiotic & Cheek & 3.5 & M & Slip down & - & Dehiscence & - & 8 \\
\hline 8 & Female & 32 & Antibiotic & Chin & 1.0 & M & Slip down & - & Dehiscence & - & 10 \\
\hline 9 & Male & 32 & Antibiotic & Forehead & 10.0 & M & Slip down & - & Dehiscence & - & 10 \\
\hline 10 & Male & 72 & Antibiotic & Forehead & 3.0 & $M$ & Sharp instrument & - & Necrosis & 20 & 10 \\
\hline 11 & Female & 23 & Antibiotic & Forehead & 7.0 & M & Slip down & - & Dehiscence & - & 8 \\
\hline 12 & Female & 82 & Antibiotic & Nose & 2.0 & $\mathrm{D}$ & Slip down & Heart failure & Necrosis & 50 & 18 \\
\hline 13 & Male & 61 & Antibiotic & Forehead & 2.5 & M & Slip down & - & Dehiscence & - & 10 \\
\hline 14 & Male & 59 & Antibiotic & Eyebrow & 3.5 & M & Unknown & Hypertension & Dehiscence & - & 10 \\
\hline 15 & Female & 87 & Antibiotic & Cheek & 8.0 & $D$ & Slip down & Hypertension & Necrosis & 40 & 24 \\
\hline
\end{tabular}

PMH, past medical history; EGF, epidermal growth factor; M, muscle rupture; SC, subcutaneous fat; B, bone exposure; D, dermis; CAOD, coronary artery occlusive disease. 
time was 12.7 days, which was longer than the overall average.

\section{Cases}

Case 1

A 47-year-old male patient was hit on the face by a can. He showed an avulsion laceration on his left cheek. The margin of the avulsed flap was slightly congestive at the time of his emergency room visit. The laceration was sutured with nylon \#6-0 and he was prescribed the antibiotic ointment. Four days after repair, necrotic change was observed; 6 days after repair, dehiscence with eschar was found after stitches were removed. Additional dressing was performed, and 24 days after repair, the wound healed completely (Fig. 1).

\section{Case 2}

An 82-year-old female patient with hypertension slipped down a slope. She suffered an avulsion laceration on her nasal dorsum without any facial bone fracture. The nearly $2 \mathrm{~cm}$ long, dermis layer-depth laceration was sutured with nylon \#6-0 and she was prescribed the antibiotic ointment. The wound was purple at the time of her visit to the emergency department, and although there was slight lightening of the flap color 5 days after repair, mild dehiscence was observed. The wound healed completely after 18 days (Fig. 2).

\section{Discussion}

The emergency room encounters a variety of laceration cases occurring due to various causes such as sharp knives or blunt force [3]. The types of lacerations vary as do the causes [4]. In some cases, damage to the surrounding tissue accompanies a blunt force or a friction force. On the other hand, injuries from
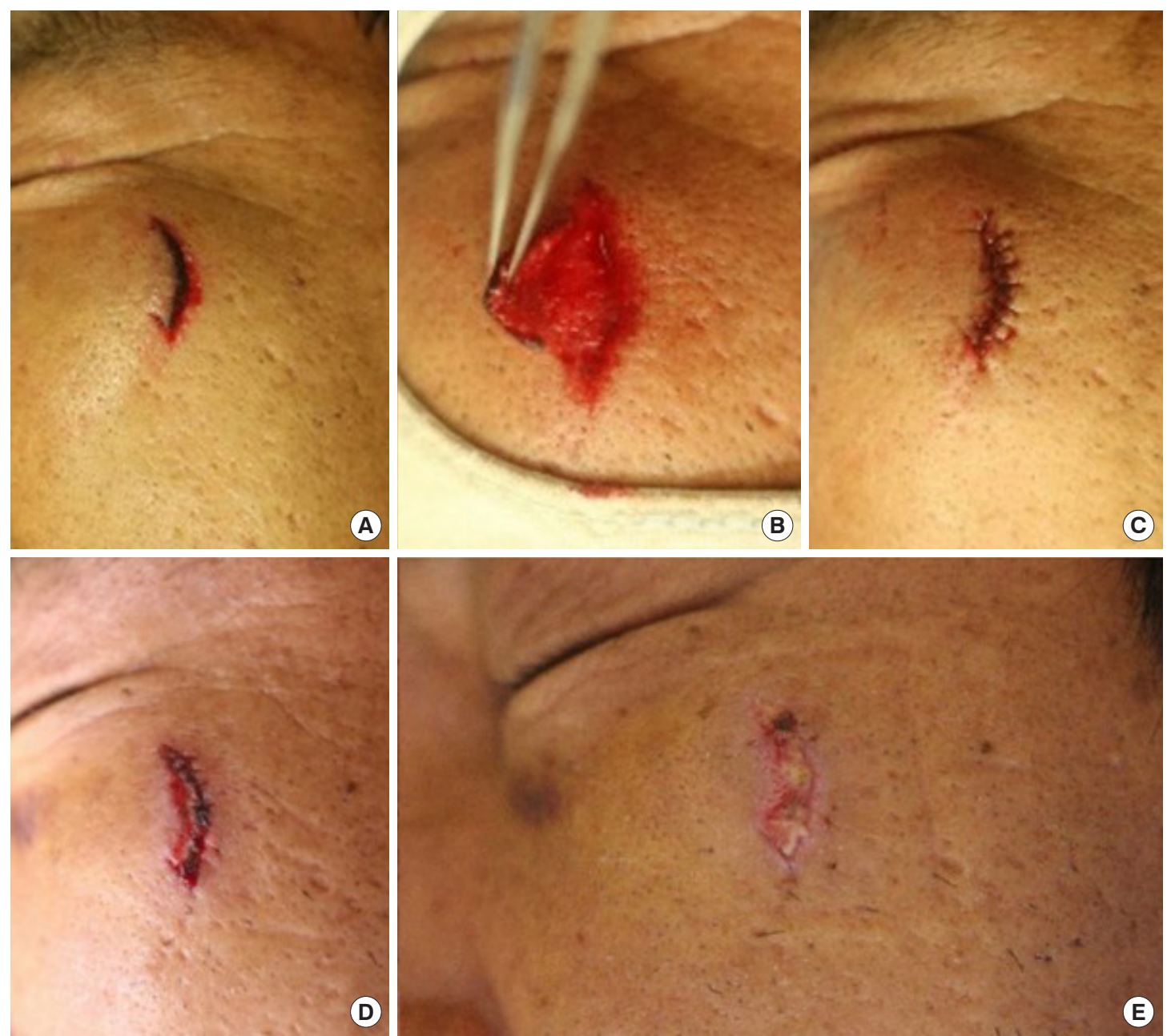

Fig. 1. Avulsion laceration on the cheek. (A, B) Avulsion laceration on the cheek at the time of emergency department visit. (C) After primary repair by suturing. (D) Necrotic change 4 days after repair. (E) Eschar with necrotic change 6 days after repair. 

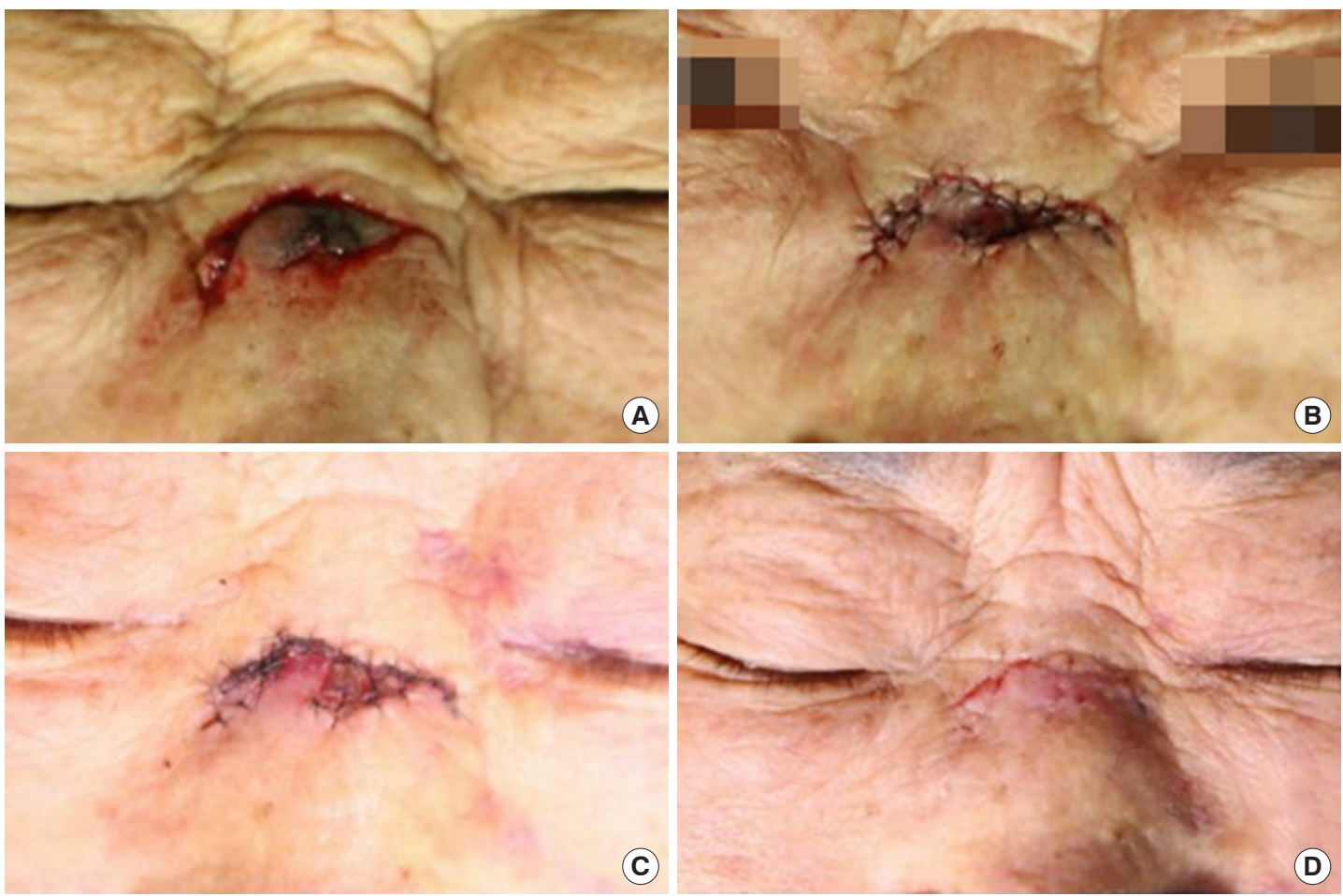

Fig. 2. Avulsion laceration on the nasal dorsum. (A) Avulsion laceration on the nasal dorsum at the time of emergency department visit. (B) After primary repair by suturing. (C) Necrotic change on wound margin 6 days after repair. (D) Healing of wound 18 days after repair.

knives are generally not accompanied by damage to surrounding tissues. The depths of lacerations vary from skin tears that detach the epidermis from the dermis to deep lacerations in the tendon, muscle, and even bone. In addition, the surface shapes of the lacerations can vary from their shapes at the deepest part of the wound. In this study, we examined lacerations that extended beyond the depth of the dermis and required suturing but no additional surgical treatment such as tenorrhaphy, neurorrhaphy, or myorrhaphy. Furthermore, the object of our study was the patients whose injuries were in an oblique line.

Avulsion lacerations caused by an oblique force usually have more complications in wound healing compared to vertical lacerations [5]. While vertical lacerations generally heal well with fine scarring, avulsion lacerations frequently heal with some complications, such as necrosis following dehiscence or infection due to unfavorable blood supply to the periphery of avulsed flap. Prolonged recovery of the avulsion laceration leads to longer periods of treatment as well as scarring, which can be critical in cases of facial lacerations.

The therapeutic principle of laceration has not changed significantly over time. Treatment focuses on preventing infec- tion, shortening the duration of treatment, and minimizing scarring. Repairs such as suturing or taping help facilitate rapid healing and prevent secondary closure, minimizing patient discomfort and scarring.

After repair, any topical ointments can help maintain moisture in the wound and prevent dressings from sticking to the wound $[6,7]$. Though it is true that topical antibiotic ointments are essential in acute infectious wounds as they act as antimicrobials [8-10], there are some reservations about the use of preventive topical antibiotics because of concerns of bacterial tolerance.

Ofloxacin-containing ophthalmic ointment has been used for a long time. It is safe for the mucosa and eyes and suitable for use on various skin wounds. Ofloxacin is a broad-spectrum synthetic fluorinated quinolone described in 1982 and can be used against Gram-positive and Gram-negative bacteria as well as skin-damaging bacteria that cause infection in laceration wounds. Moreover, it can be used for infections of the respiratory and urinary tracts, kidneys, skin, and soft tissue, etc. Topical antimicrobials can be effective, because they function in the presence of a biofilm and can help eliminate biofilms from the wound bed [6]. However, the fact remains 
that indiscriminate use of antibiotics may cause bacterial tolerance. Studies have therefore been conducted for the replacement of antibiotics [11]. Patients with infectious conditions were not included in our study, and we did not observe any difference in the levels of infection occurring with the use of the EGF ointment or the ofloxacin ointment, proving that we can cease the habitual use of preventive antibacterial ointment after initial repair.

Human EGF has been shown to be effective in the growth of mammalian cells, particularly epithelial and skin cells and in regeneration of skin injured with wounds and burns [12-14]. In 1996, recombinant human EGF-expressing strains were developed using genetic recombination techniques. Studies have been carried out on the use of the developed EGF to aid wound healing in rats and pigs, and their effectiveness has been demonstrated in several studies $[15,16]$. Repeated topical application of EGF has been shown to accelerate the healing rate of traumatic skin damage [17]. Recently, a study stated that topical application of EGF decreases the formation of excessive scar tissue [18]. In addition, local application of EGF modulates the wound tensile strength by decreasing the histamine levels in skin tissue [19]. In our study, there was no difference in necrosis incidence between patients who had been administered the antibiotic ointment or the EGF ointment. However, application of the EGF ointment resulted in significantly lower percentages of necrosis areas than when ofloxacin ointment was applied. The known regeneration capacity and wound healing effects of EGF on skin cells might explain such results.

Because complications in facial lacerations are not common, it was difficult to compare the incidence of necrosis and dehiscence in a study with such a small sample. Although the efficacy of the EGF ointment in necrosis has been shown in 119 patients, studies on its efficacy on different complications, laceration types, and locations on a larger pool of patients are needed in the future. We believe the current study provides a foundation for future research.

This study does have its shortcomings. All the wounds with complications observed in the study were small wounds, because the study dealt with wounds repaired in the emergency room, while larger wounds are typically treated in the operating room. Also, due to the original design of the study, the area of necrosis (\%) of each group was calculated as the mean value of each individual wound's necrosis percentage (including the majority of wounds which presented $0 \%$ necrosis), instead of the percentage of total necrosis area against total wound area of all wounds in each group. Further research should include larger wounds and compare the areas of complications in absolute terms instead of percentages.

A simple laceration on the face is a common reason to visit the emergency department. Most traumatic injuries heal without necrosis, dehiscence or other complications. But avulsion lacerations carry more risk in skin healing than simple vertical lacerations. This study suggests that topical treatment with EGF ointment after suturing avulsion lacerations may promote healing. We therefore recommend treatment with EGF ointment for wound healing in patients with avulsion lacerations.

\section{Conflict of interest}

Funding for this study was supported by Daewoong Pharmaceutical Co., Ltd., the manufacturer of the epidermal growth factor ointment used in the study. No other potential conflict of interest relevant to this article was reported.

\section{ORCID iD}

Youngwoong Choi https://orcid.org/0000-0002-2211-2981

\section{References}

1. Kwon YB, Kim HW, Roh DH, et al. Topical application of epidermal growth factor accelerates wound healing by myofibroblast proliferation and collagen synthesis in rat. J Vet Sci 2006;7:105-9.

2. Savage CR Jr, Inagami T, Cohen S. The primary structure of epidermal growth factor. J Biol Chem 1972;247:7612-21.

3. Hollander JE, Singer AJ. Laceration management. Ann Emerg Med 1999;34:356-67.

4. Hollander JE, Singer AJ, Valentine S, et al. Wound registry: development and validation. Ann Emerg Med 1995;25: 675-85.

5. Bilo RAC, Oranje AP, Shwayder T, et al. Cutaneous manifestations of child abuse and their differential diagnosis: blunt force trauma. Heidelberg: Springer Science \& Business Media; 2012.

6. Winter GD. Effect of air exposure and occlusion on experimental human skin wounds. Nature 1963;200:378-9.

7. Winter GD. Formation of the scab and the rate of epithelization of superficial wounds in the skin of the young domestic pig. Nature 1962;193:293-4. 
8. Desrosiers M, Bendouah Z, Barbeau J. Effectiveness of topical antibiotics on Staphylococcus aureus biofilm in vitro. Am J Rhinol 2007;21:149-53.

9. Halasz NA. Wound infection and topical antibiotics: the surgeon's dilemma. Arch Surg 1977;112:1240-4.

10. Leyden JJ, Kligman AM. Rationale for topical antibiotics. Cutis 1978;22:515-28.

11. Diehr S, Hamp A, Jamieson B, et al. Clinical inquiries: do topical antibiotics improve wound healing? J Fam Pract 2007;56:140-4.

12. Cohen S, Elliott GA. The stimulation of epidermal keratinization by a protein isolated from the submaxillary gland of the mouse. J Invest Dermatol 1963;40:1-5.

13. Brown GL, Curtsinger L 3rd, Brightwell JR, et al. Enhancement of epidermal regeneration by biosynthetic epidermal growth factor. J Exp Med 1986;163:1319-24.

14. Brown GL, Nanney LB, Griffen J, et al. Enhancement of wound healing by topical treatment with epidermal growth factor. N Engl J Med 1989;321:76-9.

15. Breuing K, Andree C, Helo G, et al. Growth factors in the repair of partial thickness porcine skin wounds. Plast Reconstr Surg 1997;100:657-64.

16. LeGrand EK, Burke JF, Costa DE, et al. Dose responsive effects of PDGF-BB, PDGF-AA, EGF, and bFGF on granulation tissue in a guinea pig partial thickness skin excision model. Growth Factors 1993;8:307-14.

17. Okumura K, Kiyohara Y, Komada F, et al. Improvement in wound healing by epidermal growth factor (EGF) ointment. I. Effect of nafamostat, gabexate, or gelatin on stabilization and efficacy of EGF. Pharm Res 1990;7:1289-93.

18. Gope R. The effect of epidermal growth factor \& plateletderived growth factors on wound healing process. Indian J Med Res 2002;116:201-6.

19. Babul A, Gonul B, Dincer S, et al. The effect of EGF application in gel form on histamine content of experimentally induced wound in mice. Amino Acids 2004;27:321-6. 